\title{
Spatial memory: behavioral determinants of persistence in the watermaze delayed matching-to-place task
}

\author{
Bruno M. da Silva, ${ }^{1,3}$ Tobias Bast, ${ }^{1,2}$ and Richard G.M. Morris ${ }^{1}$ \\ ${ }^{1}$ Laboratory for Cognitive Neuroscience, Centre for Cognitive and Neural Systems, The University of Edinburgh, Edinburgh EH8 9JZ, United \\ Kingdom; ${ }^{2}$ School of Psychology and Neuroscience@Nottingham, University of Nottingham, Nottingham NG7 2RD, United Kingdom
}

\begin{abstract}
The watermaze delayed matching-to-place (DMP) task was modified to include probe trials, to quantify search preference for the correct place. Using a zone analysis of search preference, a gradual decay of one-trial memory in rats was observed over $24 \mathrm{~h}$ with weak memory consistently detected at a retention interval of $6 \mathrm{~h}$, but unreliably at $24 \mathrm{~h}$. This forgetting function in the watermaze was similar to that found using a search-preference measure in a food-reinforced dry-land DMP task in a previous study. In a search for strong and weak encoding conditions, essential for a later behavioral tagging study, three encoding trials gave strong 6-h and 24-h memory when trials were separated by 10 min (spaced training) but not $15 \mathrm{sec}$ (massed training). The use of six encoding trials gave good 6-h memory with both spaced and massed training. With respect to weak encoding, placement on the escape platform, instead of the rat swimming to it, resulted in detectable memory at $30 \mathrm{~min}$ but this had faded to chance within $24 \mathrm{~h}$. In contrast to the search-preference measure, latencies to cross the correct place revealed neither the gradual forgetting of place memory nor the benefit of spaced training.
\end{abstract}

Place memory has long been studied in rodents to investigate the psychological processes and neurobiological substrates of declarative memory (O'Keefe 1976; Morris et al. 1982; Aggleton and Pearce 2001). Place memory, as a subcomponent of declarative memory, relies on the rapid encoding of allocentric relations among multiple cues such that goals can be approached from different positions. A number of brain structures are involved in encoding, storage, and consolidation of place memory, including the hippocampus and its interactions with parahippocampal cortices, the diencephalon, and various regions of the prefrontal and midline cortices (O'Keefe and Nadel 1978; Sutherland et al. 1983, 1988; Sutherland and Rodriguez 1989; Squire 1992; Eichenbaum 2000; Leutgeb et al. 2005; Aggleton et al. 2010; Wang and Morris 2010; Euston et al. 2012). However, after the encoding of a new spatial memory representation or "engram," what are the determinants of how long such a memory may last?

The general view, from study of many different forms of learning, is that both the number and spacing of learning trials are important for memory persistence (Ebbinghaus 1885; McGaugh 1966), as are the amount and timing of reinforcement (Rescorla 1989). However, place memory is sometimes considered distinct with, for example, the cognitive-map theory asserting that learning can occur in a single trial and, apparently, independently of reinforcement (O'Keefe and Nadel 1978). Even if this is the case, place learning is likely to be subject to "modulation" as are other forms of learning. Reinforcement of learning has long been linked to dopamine (Wise 2004). Lisman and Grace (2005) have suggested that the likely sensitivity of hippocampal-dependent learning to modulation by unexpected novelty could be mediated by dopaminergic activation of the hippocampus from the ventral tegmental area (VTA). Similarly, the synaptic tagging and capture (STC) theory (Frey and Morris 1998; Redondo and Morris 2011) asserts that events before and after encoding that up-regulate the availability of plasticity-related proteins (PRPs) can extend the persistence of memory traces, possibly via the very mechanism out-

\footnotetext{
${ }^{3}$ Corresponding author

E-mail brunofts@gmail.com

Article is online at http://www.learnmem.org/cgi/doi/10.1101/Im.032169.113.
}

lined by Lisman et al. (2011) in a revision of their theory. The present work was conducted with a view to a behavioral project looking at the functional impact of STC, sometimes called "behavioral tagging" (Moncada and Viola 2007; Ballarini et al. 2009; Wang et al. 2010; Moncada et al. 2011), the focus here being to establish baseline forgetting functions for rapidly acquired place memory against which manipulations that might enhance memory persistence could later be compared. However, the manipulations and findings also have broader relevance to a variety of different ideas about memory modulation distinct from STC.

We used the delayed matching-to-place (DMP) task, which is an unusual variant of the watermaze protocols in which rats (or mice) learn to escape to a hidden platform that is typically moved to a new location each day and performance is followed across many days and weeks (Morris 1983, 1984; Panakhova et al. 1984; Whishaw 1985; Steele and Morris 1999; Chen et al. 2000; Nakazawa et al. 2003; O'Carroll et al. 2006). Escape efficiency depends on the rapid acquisition and subsequent retrieval, up to several hours later, of allocentric place memory that is expressed as successful escape to the new location from any starting point. Importantly, there is no necessity for long-term systems consolidation for effective performance, as new learning occurs each day against a backdrop of unchanging context information. A human analogy might be that of a rail commuter who tends to go to work a bit later than others and has therefore, each day, to find a spare parking slot at the station car park. The commuter must remember where the car was parked that day when returning from work, and remembering over days is not necessary (although it may occur). A key feature of such "everyday" memory (Wang et al. 2010) is the possibility to repeatedly test the formation and retention of new place memories in the same rat using a withinsubjects design (Steele and Morris 1999; O'Carroll et al. 2006; Pezze and Bast 2012). The DMP task is very sensitive to disruption

\footnotetext{
(C) 2013 da Silva et al. This article is distributed exclusively by Cold Spring Harbor Laboratory Press for the first 12 months after the full-issue publication date (see http://learnmem.cshlp.org/site/misc/terms.xhtml). After 12 months, it is available under a Creative Commons License (AttributionNonCommercial 3.0 Unported), as described at http://creativecommons. org/licenses/by-nc/3.0/.
} 
of hippocampal function (Steele and Morris 1999; Ferbinteanu et al. 2003; Nakazawa et al. 2003; de Hoz et al. 2005; O'Carroll et al. 2006; Bast et al. 2009; Pezze and Bast 2012).

Search preference, as measured on probe trials when the platform is unavailable, has long been recognized as the most reliable and sensitive measure of allocentric place memory in referencememory versions of the watermaze task, in which the platform location remains constant across trials and testing days (e.g., Morris 1981; Buresova et al. 1985; Schenk and Morris 1985; Moser et al. 1998). We recently found a monotonic decline of one-trial place memory, as measured using a probe test measure of search preference, using a dry-land food-reinforced DMP task in which rats learn to dig for food in a sandwell that is moved to a novel location each day in an event arena (Bast et al. 2005). This monotonic decline was observed with retention intervals after encoding ranging from a few seconds to $6 \mathrm{~h}$. In contrast, no consistent decline in memory strength has yet been found with increasing retention intervals in the aversively motivated watermaze DMP task (Morris et al. 1990; Steele and Morris 1999; von Linstow Roloff et al. 2002; O'Carroll et al. 2006; but see Panakhova et al. 1984; de Hoz et al. 2005). Although the difference in sensitivity to retention interval may be due to differential motivation (Bolhuis et al. 1985), a likely alternative is the need to use a sensitive probe test measure to see such a function clearly. The original versions of the DMP task measured rapid place learning as a reduction in escape latencies or path lengths across successive trials to a new platform location each day, with performance typically averaged across days. However, these measures display variability due to occasional chance findings of the hidden platform, and they may also be influenced by systematic search strategies and/or the use of single beacon cues (e.g., Morris 1981; Buresova et al. 1985; Schenk and Morris 1985; Jacobs and Schenk 2003).

We therefore developed a modification of the watermaze DMP protocol that includes probe trials, during which the escape platform is sometimes withheld for a period of $60 \mathrm{sec}$, so that search preference can be measured and used as an index of rapidly acquired place memory (zone analysis) (Bast et al. 2009; Jackson et al. 2011; Pezze and Bast 2012) (Fig. 1A,B). After $60 \mathrm{sec}$, the so-called "Atlantis platform" (Spooner et al. 1994) rises from the bottom of the pool enabling rewarded escape even on probe trials. Our focus was on whether such one-trial encoding of memory results in traces that display time-dependent forgetting (Experiment 1) and, if so, whether and how the strength of memory traces is affected by the type, number, and temporal distribution of multiple encoding trials (Experiments 2-4). Finally, we compare performance in this "benchmark" task against that observed in a separate dry-land "event-arena" task reported earlier (Bast et al. 2005).

\section{Results}

\section{Experiment 1: forgetting of one-trial place memory}

In Experiment 1, we investigated if a search-preference measure on occasional probe tests would reveal a monotonic timedependent decay of one-trial place memory in the watermaze.

A common cohort of male Lister hooded rats $(n=20)$ was used in each of three replications. The retention intervals between encoding and retrieval trials were $15 \mathrm{sec}, 15 \mathrm{~min}, 30 \mathrm{~min}$, and $1 \mathrm{~h}$ in replication $1 ; 15 \mathrm{sec}, 1 \mathrm{~h}, 3 \mathrm{~h}$, and $6 \mathrm{~h}$ in replication 2 ; and $6 \mathrm{~h}$ and $24 \mathrm{~h}$ in replication 3 . Retention intervals of $15 \mathrm{sec}, 1 \mathrm{~h}$, and $6 \mathrm{~h}$ were included across these replications to examine performance stability (Strijkstra and Bolhuis 1987) and so enable the overall analysis to include all retention intervals.

We first trained the rats for $8 \mathrm{~d}$ using the standard protocol of the DMP watermaze task (Steele and Morris 1999), which involves 
four trials/day to a platform in the same location throughout the day, with a change to a novel platform location at the beginning of each day (Fig. 1A). Escape latency on trial 1 (T1) is generally long-as even an experienced rat has no way of knowing where it is located-but good performance on trial 2 of each day reflects retrieval of memory that has been rapidly encoded during trial 1. Trials 3 and 4 are scheduled only to reinforce the "win-stay" rule of the task. For days 1-4 of training, the inter-trial interval (ITI) was about $15 \mathrm{sec}$ between all trials. For days $5-8$, the ITI between trial T1 and T2 was varied between $15 \mathrm{sec}, 1 \mathrm{~h}, 3 \mathrm{~h}$, and $6 \mathrm{~h}$ $(\triangle \mathrm{RI}$ in Fig. $1 \mathrm{~A})$ in order to familiarize the rats with varying retention intervals (the order of assignment was counterbalanced across intervals with each retention interval used on each day for a different quarter of the rats). On training days, the platform was hidden just underneath the water surface during the whole trial duration, so that rats could climb on the platform as soon as they reached the correct location. The impact of changing retention interval between trial 1 (encoding) and trial 2 (retrieval) on the strength of one-trial place memory during retrieval was tested on selected probe days. On these, the Atlantis platform was held at the bottom of the pool for $60 \mathrm{sec}$ and then raised, so that search preference for the correct location on trial 2 could be measured as "percentage of time in correct zone" using the zone analysis (Moser et al. 1998). More specifically, to measure search preference for the vicinity of the platform location, eight $40-\mathrm{cm}$ diameter "virtual" zones were defined across the pool surface, so that one zone, the "correct zone," was concentric with the platform location (12-cm diameter), and all eight zones were nonoverlapping, evenly spaced, and symmetrically arranged (Fig. 1B). The time spent in each of these eight zones during the 60 -sec probe trial was measured, and from these measures the "percentage of time in correct zone" was calculated as: ([time in correct zone/total time in all eight zones] $\times$ 100) (Bast et al. 2009; Jackson et al. 2011; Pezze and Bast 2012).

\section{Training}

Latency data during the eight initial training days is shown in Figure 1C. Latency was highest on trial 1, reflecting search for the new daily platform location, with a sharp reduction from trial 1 to trial 2, reflecting one-trial learning. This characteristic pattern was evident from the start of training, with latencies on trials 2-4 consistently $<20 \mathrm{sec}$ from the seventh day as in previous studies (Steele and Morris 1999; O'Carroll et al. 2006; Bast et al. 2009; Pezze and Bast 2012). Training then continued from day 9 to day 28, with 10 probe tests and 10 interleaved training days. Average daily performance across this interleaved training is shown in Figure 1D.

\section{Probe days: memory decline with increasing retention intervals}

The probe test zone-analysis data from the three replications was combined as analysis of the performance on the overlapping retention intervals (15 sec, $1 \mathrm{~h}$, and $6 \mathrm{~h}$ ) revealed no differences $(F<1)$. Our first key finding is that percentage of time searching in the correct zone declined monotonically with increasing retention interval after one-trial encoding $\left(F_{(6,114)}=10.0, P<0.0001\right)$ (Fig. 1E). Post-hoc Fisher's least significant difference (LSD) comparisons indicated that performance at the 15-sec retention inter$\operatorname{val}(36.0 \pm 2.3 \%$ relative to the $12.5 \%$ chance level for eight zones) was higher than at all other intervals $(P<0.005)$. Search preference for the correct zone was also found to be greater at $15 \mathrm{~min}$ than at both $6 \mathrm{~h}(P<0.05)$ and $24 \mathrm{~h}(P<0.0005)$; at $30 \mathrm{~min}$ relative to $6 \mathrm{~h}(P<0.005)$ and to $24 \mathrm{~h}(P<0.0005)$; and stronger at a retention interval of $1 \mathrm{~h}$ as compared to $3 \mathrm{~h}$ and $24 \mathrm{~h}(P<0.05$ and $P<0.01$, respectively). Search preference was also above chance at all retention intervals up to $6 \mathrm{~h}\left(t_{(19)}=5.2, P<0.0001\right)$, but no longer at $24 \mathrm{~h}\left(15.7 \pm 2.0 \%, t_{(19)}=1.5, P=0.14\right)$.

We also measured T2 latencies. The first crossing latencies for T2 showed a less consistent and nonmonotonic sensitivity to retention interval than the zone analysis for search preference (Fig. 1F). A repeated-measures analysis of variance (ANOVA) of T2 latencies did reveal a modest but significant effect of retention intervals $\left(F_{(6,114)}=2.2, P<0.05\right)$, but the fastest escape on T2 was at the 30-min retention interval. Post-hoc Fisher LSD comparisons of T2 latencies revealed faster crossing of the correct location at 30 min compared to $3 \mathrm{~h}(P<0.05), 6 \mathrm{~h}(P<0.005)$, and $24 \mathrm{~h}(P<$ $0.05)$. The T2 latencies for $15 \mathrm{sec}$ and $15 \mathrm{~min}$ were both faster than at $6 \mathrm{~h}(P<0.05)$, but not at the other intervals.

Analysis of latency savings (i.e., latency reduction from T1 to T2) did not reveal an effect of retention interval on performance $\left(F_{(6,114)}=2.0, P=0.07\right.$; data not shown). The primary reason for this was because of substantial variation in T1 latency from which the T2 latency is subtracted. This is unfortunate for it is clearly sensible to ask, using a latency savings measure, how much quicker a rat is to escape over training within a day. However, using the subtraction of two latency measures, each subject to chance factors, actually builds in greater variability, precluding its use as a measure.

Collectively, these results suggest that memory of an escape location learned in one trial decays monotonically from a relatively strong memory up to $30 \mathrm{~min}$ after encoding to a weak but detectable memory at $6 \mathrm{~h}$, and then its loss, or near loss, by 24 h. Our data also show that the search preference measure is particularly sensitive in revealing a monotonic decline of memory, with an $F$ value of 10.0 , whereas T2 latencies, while showing a significant effect of retention interval, nonetheless showed greater variability. The savings measure did not reveal statistically significant dependence on the retention interval at all. These data were secured against a background of DMP task stability over time with high retest reliability across replications for the common retention intervals as assessed by zone analysis.

\section{Experiments 2 and 3: facilitation of long-term retention by repetition and spacing of encoding trials}

Additional trials and spaced training facilitate memory in a variety of species, including humans (Ebbinghaus 1885) and rodents (McGaugh 1966; Domjan 1980; Roberts and Dale 1981; Fanselow and Tighe 1988; Genoux et al. 2002; Scharf et al. 2002). In the watermaze also, spaced training improves long-term place memory when reference memory training occurs over days (Morris and Doyle 1985; Kogan et al. 1997; Spreng et al. 2002; Bolding and Rudy 2006; Sisti et al. 2007).

To examine the impact of number and spacing of learning trials in the DMP task, trial 1 (encoding) of the basic protocol used in Experiment 1 was replaced by three or six memory encoding trials at varying spacing. We can think of these as Trials $1_{1}, 1_{2}, 1_{3}$, etc. followed by the trial in which the impact of this different pattern of encoding is assessed-this being always designated as trial 2 . This terminology may seem confusing as "trial 2 " could then be the fourth or seventh trial of the day, but we have found it easier to think of "trials 1 " as encoding, and "trial 2" as memory retrieval.

A new cohort of male Lister hooded rats $(n=18)$ was used for Experiment 2, and another batch $(n=16)$ for Experiment 3.

\section{Experiment 2: time on platform after escape and the number and spacing of memory encoding trials}

Experiment 2 investigated the impact of manipulations at encoding (Fig. 2A): (1) variation of time on the hidden platform after 
A Experimental conditions

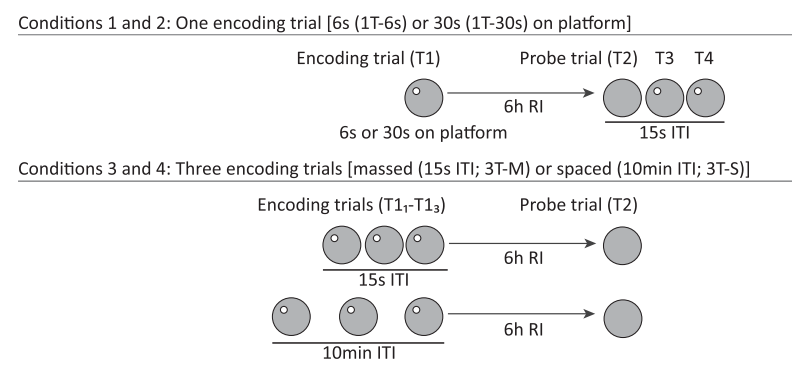

Conditions 5 and 6: Six encoding trials [massed (15s ITI; 6T-M) or spaced (10min ITI; 6T-S)]

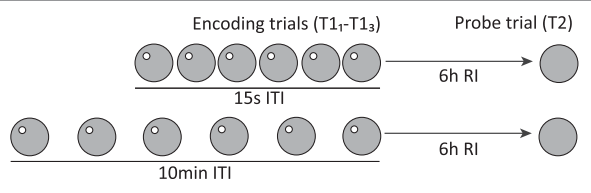

B Zone analysis

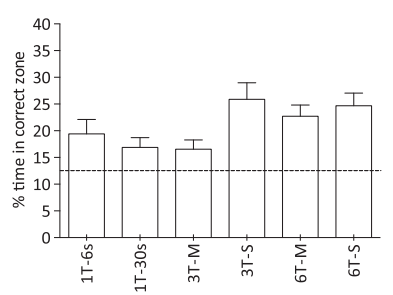

C Latencies

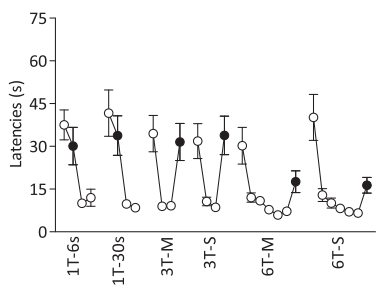

Figure 2. Variation in long-term place memory $(6 \mathrm{~h})$ as a function of time on platform, spacing, and repetition of acquisition trials (Experiment 2, $n=18$ ). (A) Variation in conditions $1-6$ reflect time on the platform (1T-6 sec or 1T-30 sec), massed or spaced encoding trials $\left(\mathrm{T} 1_{1}-\mathrm{T} 1_{3}\right)$ separated by $15-\mathrm{sec}$ or $10-\mathrm{min}$ ITI (3T-M or 3T-S), and additional repetition of encoding trials $\left(\mathrm{T} 1_{1}-\mathrm{T} 1_{6}\right)$ separated by $15-\mathrm{sec}$ or 10-min ITI (6T-M or 6T-S). (B) Percentage of time in correct zone during T2 probe trials. Note striking effect of trial spacing when three encoding trials are given. Stippled horizontal line indicates chance value for percentage swim time in correct zone. (C) Latencies. Black circles represent retention trial (T2) latencies. Means \pm 1 SEM.

escape from the water $(6 \mathrm{sec}$ and $30 \mathrm{sec}$; in our watermaze experiments, including the other experiments in this study, the time we usually allow rats to spend on the platform after escape is $30 \mathrm{sec}$ ); (2) increasing the number of encoding trials (one, three, and six), and (3) varying ITI during multitrial encoding (15 sec vs. $10 \mathrm{~min}$ ). All conditions were tested in a within-subjects design extending over $18 \mathrm{~d}$ (after 10 training days). With respect to (1), variation in the time that rats spend on the escape platform, there is evidence that a longer escape period may enable rats to acquire more information about the relative position of the platform in relation to environmental cues (Sutherland and Linggard 1982; Keith and McVety 1988; Whishaw 1991). It is also known that the use of multiple encoding trials and a longer time between trials, e.g., $10 \mathrm{~min}$, facilitates the formation of long-term memory in conventional multitrial long-term memory tasks (e.g., Fanselow and Tighe 1988; Kogan et al. 1997; Josselyn et al. 2001; Genoux et al. 2002); it is also the protocol of choice for the induction of long-lasting protein synthesis-dependent forms of hippocampal synaptic plasticity in vitro by repeated synaptic stimulation (e.g., Reymann et al. 1985; Frey et al. 1993; Scharf et al. 2002). We wondered if these conditions may also constitute a "strong" encoding condition for the DMP task.

During the initial training on the task (10 d), before comparing performance between the experimental conditions, we first established that a single encoding trial would result in comparable and reproducible levels of memory at the 6-h retention interval.
Search preference on trial 2, run as a probe with the Atlantis platform at $6 \mathrm{~h}$ after trial 1 on days 7 and 10 of training, confirmed that this was the case. The average percentages of time in the correct zone were $17.5 \pm 2.2 \%$ on day 7 and $16.1 \pm 1.4 \%$ on day 10 (chance $=12.5 \%$ ), which are above chance and similar to the results for the 6-h retention interval in Experiment 1.

An ANOVA of zone-analysis scores for the series of probe tests for the Experiment 2 revealed a highly significant difference between parametric conditions $\left(F_{(5,85)}=3.6, P<0.01\right)$ (Fig. 2B). Post-hoc Fisher's LSD tests were then used to examine the separate conditions. (1) Impact of time on the platform-no significant difference was observed in performance after rats were allowed 6 sec or $30 \mathrm{sec}$ on the platform in a single encoding trial $(P=0.4)$. (2) Impact of varying number of encoding trials-six massed trials produced stronger memory than three massed trials $(P<0.05)$, but six spaced trials showed only a nonsignificant trend toward stronger memory than three spaced trials $(P=0.07)$. This may imply that performance had reached ceiling levels with three spaced trials. (3) Impact of varying the temporal spacing of encoding trials-three spaced trials produced stronger memory than three massed trials $(P<0.005)$, but six spaced trials did not produce stronger memory than six massed trials $(P=0.51)$. Once again, performance had likely reached ceiling levels after three spaced encoding trials. (4) Synergistic effects between number and interval of encoding trials-performance after one encoding trial (30 sec on platform) was weaker than performance obtained after three $(P<0.01)$ and six $(P<0.05)$ spaced trials, but not statistically different from performance obtained after three $(P=0.91)$ or six $(P=0.05)$ massed trials. Finally, the zone analysis revealed above-chance performance for all conditions (the least significant being the three massed trial condition, $\left.t_{(17)}=2.4, P<0.05\right)$.

We also analyzed retention trial (T2) latencies but not latency savings (the latter was excluded because the choice of start positions in the watermaze prevented proper counterbalancingthese positions were adjusted on probe test days to ensure that the sequence of them between the last encoding trial (i.e., $\mathrm{T} 1_{1}$, $\mathrm{T} 1_{3}$, or $\mathrm{T} 1_{6}$ ) and the retention trial (T2) was always the same). An overall ANOVA of T2 latencies showed a just significant difference between conditions $\left(F_{(5.85)}=2.4, P<0.05\right)$ (Fig. 2C), but the post-hoc Fisher's LSD tests revealed only an enhancing effect of the number of encoding trials on memory at $6 \mathrm{~h}$. Neither time allowed on the platform at the end of the encoding trial nor the spacing of encoding trials seemed to affect latencies on retention trials. Data from these conditions were therefore pooled to conduct a separate ANOVA of trial number $\left(F_{(2.34)}=6.39, P<\right.$ $0.005)$, with post-hoc tests showing reduced latency at retrieval after six relative to after one or three encoding trials $(P<0.005)$.

\section{Experiment 3: comparison of memory at $6 \mathrm{~h}$ and $24 \mathrm{~h}$ for one and three spaced encoding trials}

For the impending "behavioral tagging" experiments, it is essential to have a strong encoding condition that results in good memory after a long memory interval and a weak encoding condition in which initially detectable memory falls to chance. Accordingly, it became of interest to explore memory tests after $6 \mathrm{~h}$ or after 24 h. A separate cohort of experimentally naive rats $(n=16)$ was used to compare memory at these retention intervals after either onetrial or three spaced-trial encodings, the aim being to see if these could serve as the weak and strong encoding conditions, respectively. In the process we sought also to replicate the finding of Experiment 2 that three spaced encoding trials produce stronger memory at a 6 -h retention interval than a single encoding trial, and investigate if this difference could also be observed at $24 \mathrm{~h}$.

Following initial training, a series of interleaved training and probe tests was conducted. This study showed, using the preferred 
zone analysis, that three spaced encoding trials produce better memory at $6 \mathrm{~h}$ relative to one-trial encoding $\left(F_{(1,15)}=8.4, P<\right.$ 0.01) (Fig. 3A), replicating the findings of Experiment 2 (Fig. 2B). Search preference for the correct zone was above chance in both conditions $\left(t_{(15)}=5.1, P<0.0005\right)$. T2 latencies did not differ significantly between the two conditions $\left(F_{(1.15)}<1\right)$ (Fig. 3B). At $24 \mathrm{~h}$, a similar pattern of results was observed (zone analysis, $F_{(1.15)}=6.7, P<0.05$ [Fig. 3C]; retention trial latencies, $F_{(1.15)}=$ $3.9, P>0.05$ [Fig. 3D]). Surprisingly, search preference indicated above-chance performance after a single encoding trial (18.3 \pm $\left.1.5 \%, t_{(15)}=3.8, P<0.005\right)$ as well as after three spaced encoding trials as expected $(23.1 \pm 1.5 \%)$. This suggests that an ostensibly weak one-trial memory can occasionally be still detected $24 \mathrm{~h}$ after acquisition.

\section{Experiment 4: impact of using platform placement relative to swimming on place memory at 30-min and 24-h retention intervals}

Experiments 1-3 have laid the ground work for a later "behavioral tagging" experiment, excepting that a condition in which memory is initially detectable but then falls reliably to chance has not been found. Memory for one single encoding trial was not observed after a 24-h retention interval in Experiment 1, but was seen in Experiment 3. Experiment 4 was conducted to investigate the impact of "placement" rather than swim trials-the expectation being that memory would be weaker (based on pilot observations in the laboratory). The focus was on whether we would see successful memory at $30 \mathrm{~min}$ with three spaced placement trials (trials in which rats were simply placed on the platform for 30 sec without having to swim to it) and then the loss of this memory at a 24-h retention interval (Fig. 4A). The study afforded a further opportunity to replicate the impact of three spaced swim trials at these retention intervals.

Analysis of search preference during probe trials (Fig. 4B) revealed that the animals spent more time searching the correct zone when given swim trials than when given placement trials $\left(F_{(1.15)}=17.4, P<0.001\right)$, and memory for both types of encoding event was stronger at $30 \mathrm{~min}$ than at $24 \mathrm{~h}\left(F_{(1.15)}=17.9, P<\right.$ 0.001). Even though no interaction was observed between trial type and retention interval $\left(F_{(1.15)}=3.4, P=0.09\right)$, it is noteworthy that memory was at chance $24 \mathrm{~h}$ after placement. Comparing the 30-min and 24-h retention delay, the time rats spent searching in the correct zone decreased about $15 \%$ after swim trials (a similar decline was observed with a single encoding trial [see Fig. 1E]) and 10\% after placement trials. Above-chance performance was observed for swim trials at both retention intervals $\left(t_{(15)}=3.2, P<0.01\right)$, but for placement trials memory
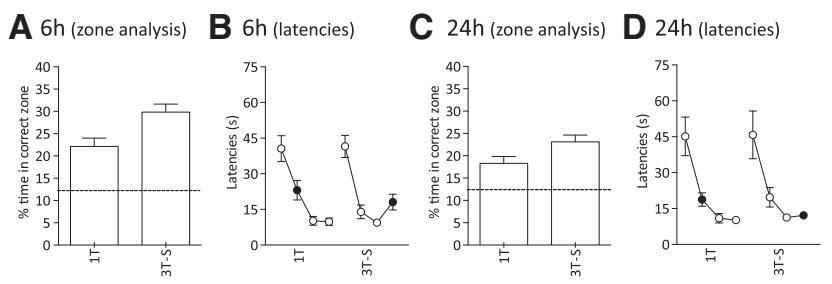

Figure 3. Enhancement of long-term place memory ( $6 \mathrm{~h}$ and $24 \mathrm{~h}$ ) by repetition and spacing of acquisition trials (Experiment $3, n=16$ ). Memory on T2 after single or three spaced (10 min ITI) encoding trials (1T or 3T-S) at $6 \mathrm{~h}(A, B)$ or $24 \mathrm{~h}(C, D)$ after encoding. $(A, C)$ Percentage of time in correct zone during probe trials. Stippled horizontal line indicates chance value for percentage swim time in correct zone. $(B, D)$ Latencies (seconds). Black circles represent retention trial latencies. Means \pm 1 SEM.
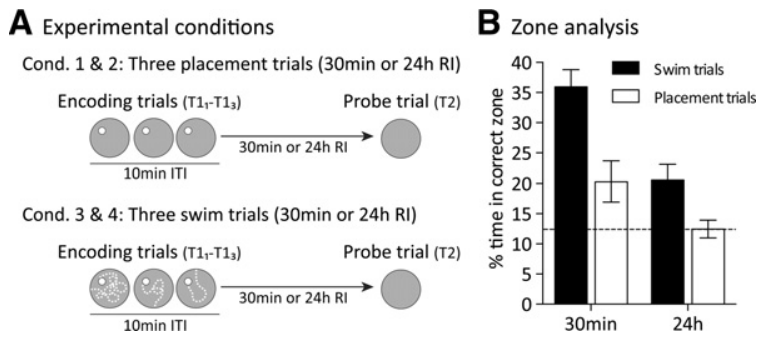

Figure 4. Optimum encoding conditions for the formation of short- or long-term place memory in the DMP watermaze protocol (Experiment 4, $n=16$ ). (A) There were three placement or three swim encoding trials separated by $10-\mathrm{min} \mathrm{ITI}\left(\mathrm{T1}_{1-3}\right)$. During each placement trial, the rats were placed on the platform for $30 \mathrm{sec}$ without swimming to it. (B) Retention (T2) measured short-term and long-term memory (30 min and $24 \mathrm{~h}$ ) after encoding as percentage of time spent searching the correct zone. Stippled horizontal line indicates chance level. Means \pm 1 SEM.

was only detectable at $30 \mathrm{~min}\left(t_{(15)}=2.3, P<0.05\right)$, but not after $24 \mathrm{~h}\left(t_{(15)}<1\right)$. The latency measure failed to reveal a main effect of retention interval on performance $\left(F_{(1.15)}=1.5, P=0.24\right.$; data not shown). Thus, overall, Experiment 4 established three spaced swim trials and three spaced placement trials as strong and weak encoding events, respectively, that could be used in future experiments to investigate tagging-like behavioral interactions.

\section{Discussion}

These experiments, using a highly sensitive probe test measure of search preference within a spatial task in which new learning occurs each day, define a set of parametric manipulations that modulate memory persistence. These may be useful for future studies examining memory enhancing and impairing manipulations. The main findings are that (1) in the DMP task, the persistence over time of spatial memory that is updated daily declines monotonically as a function of retention interval; (2) even a short period on the escape platform (6 sec) is sufficient for rapid information acquisition given that the rats will have spent longer in the watermaze swimming to this location; (3) increasing the number of encoding trials (from one to three or six) augments the reliability of persistence of memory to at least $24 \mathrm{~h}$, with three spaced trials reaching the daily asymptote; (4) increasing the spacing of multiple encoding trials (from a "massed" protocol with 15-sec inter-trial intervals to a "spaced" protocol with 10-min inter-trial intervals) also enhances memory persistence; and (5) placing the rats on the platform rather than swimming trials enables memory encoding that can be reliably detected for a short time but is clearly at chance by $24 \mathrm{~h}$. Thus, for subsequent memory modulation studies (including behavioral tagging), a "weak" memory encoding condition could be three placement trials whereas a "strong" encoding condition could be three or six swim trials.

\section{Persistence of place memory}

One-trial place memory strength declines monotonically with increasing retention intervals (up to $24 \mathrm{~h}$ ) in both the aversively motivated watermaze and in the food-reinforced event arena DMP task (Bast et al. 2005; Wang et al. 2010; this study). This is evident here with the zone analysis (watermaze) and, in the event arena, with the corresponding dig-time measure of search preference (see Fig. 5). These results do not support the view that the nature of the motivation (appetitive vs. aversive) causes differential forgetting on allocentric place memory tasks (Bolhuis et al. 1985). In both tasks, memory was shown to decay over minutes to hours 


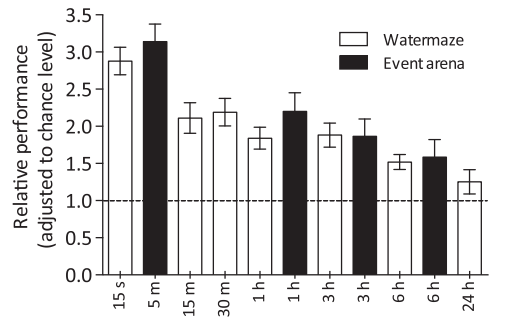

Figure 5. Comparable monotonic rates of forgetting of one-trial place memory in the watermaze and the event arena DMP tasks. Normalization of the watermaze data to chance was calculated as the percentage of time swimming in the correct zone divided by the chance level $(12.5 \%$, eight zones). Normalization of the event arena data was calculated as the percentage of time digging in the correct sandwell divided by the chance level (20\%, five sandwells; based on data of Bast et al. [2005]). Stippled horizontal line indicates chance value of normalized performance measures. Means \pm 1 SEM.

after encoding, but weak memory could sometimes, but unreliably, be seen after $24 \mathrm{~h}$.

In a rare study to examine forgetting after very short periods of training, Bolding and Rudy (2006) observed that memory for 10 consecutive trials in a watermaze (with a 2-min inter-trial interval) was detectable up to $4 \mathrm{~h}$ later when a quadrant measure of search preference was used, but only up to $30 \mathrm{~min}$ with a "difference score index of selective search" (which compares the time rats spent in the target quadrant with that spent in the second most preferred quadrant). At first glance, these results may seem to contrast with the forgetting rates observed in our study. Indeed, by using the search preference measure of performance we have shown that even a single trial produces memory that is detectable up to $24 \mathrm{~h}$ later. This may be due to the sensitivity of the performance measures used in our study, namely the zone analysis, and/or other differences in methodology. For example, rats were trained in the present task for at least $8 \mathrm{~d}$ prior to testing, whereas Bolding and Rudy's (2006) protocol consisted of a total of 10 trials all completed within a single day. This is an important difference as training over many days gives rats the opportunity to master both the contextual and procedural (nonspatial) requirements of the task and allows for a reduction in stress responses (Aguilar-Valles et al. 2005) that may sometimes be responsible for impairments of memory retention (de Quervain et al. 1998; Luksys et al. 2009).

Our focus was on finding a relatively "pure" measure of the impact of new spatial memory encoding within a context that had over days become familiar (i.e., place in context encoding). Comparison of the impact of massed vs. spaced encoding trials revealed that spacing improves memory strength with three encoding trials but apparent saturation of spacing effects thereafter. Current accounts of the impact of spaced training include the possibility of allowing for greater "consolidation" between and after trials associated with the up-regulation of plasticity-related proteins (Kogan et al. 1997; Josselyn et al. 2001; Genoux et al. 2002; Scharf et al. 2002). That spatial memory in the watermaze is sensitive to the usual parameters that affect the encoding and consolidation of other forms of memory-number and spacing of trials-indicates that spatial memory is not quite "all-or-none," as cognitive-mapping theory once held (O'Keefe and Nadel 1978).

\section{Search preference as a sensitive index of rapidly encoded place memory}

We observed differential sensitivity of the three measures of performance used to measure spatial memory strength and persis- tence. Although measures of search preference revealed rapid forgetting in the DMP task, other measures, such as first crossing latency and latency savings, showed greater statistical variability. Different measures also showed differential variability in the event arena (Bast et al. 2005). For all retention intervals, the measure of performance showing the least variability was the zone analysis, followed by retention trial (T2) latencies, and, finally, escape latency savings. The higher variability in T2 latencies and escape latency savings may have been introduced by chance factors, e.g., when the rat unexpectedly "bumps" into the platform (or "correct zone" on probe trials), with the additional variability of savings most likely resulting from the fact that savings also depend on the highly variable escape latencies during encoding trials when rats are searching for an unknown platform location. Another issue concerning escape latencies and path lengths is that they may be efficiently reduced through systematic search strategies and the use of beacon cues (e.g., Morris 1981; Buresova et al. 1985; Schenk and Morris 1985; Jacobs and Schenk 2003). In the event arena task, the higher variability of first choices and errors may explain their lower sensitivity to variations in memory strength. Chance factors contribute to such variability, such as when the rat runs into the sandwells that are closer to the start box.

Overall, these results suggest that the use of different behavioral measures may explain the varied results reported with respect to the persistence of one-trial place memory in the watermaze (Panakhova et al. 1984; Morris et al. 1990; Steele and Morris 1999; von Linstow Roloff et al. 2002; de Hoz et al. 2005). Although the results of a parametric study such as this may not seem exciting or novel, the essence of science is quantification, and the identification of search preference as an apparently optimum measure of performance in the watermaze DMP task is essential groundwork for subsequent studies of memory modulation.

\section{Characterization of encoding events and behavioral tagging}

A "behavioral tagging" study is a major undertaking as it could involve measuring gene activation, local pharmacological treatments, or even rapid reversible genetically induced changes in the hippocampus and/or other brain structures as interventions using a within-subjects protocol that also combines weak and strong encoding events. Further, the synaptic tagging and capture theory makes differential predictions about the impact of treatments at the time of spatial memory encoding (tag-setting) and later neuromodulation (modulation of plasticity-related protein [PRP] synthesis). The overall aim would be to couple weak memory encoding (as the tag-setting event) with strong memory encoding (as the up-regulator of PRPs), but under circumstances that minimize informational overlap of the two daily learning events. This, in turn, requires the use of two watermazes with different cues, and thus a new protocol that will build from the data presented here on behavioral procedures that reliably produce weak decaying spatial memory and strong persistent memory.

For now, it is important to recognize that it is the modulation of the memory of the weakly encoded event that would be the aim of such a protocol. Placement of the rat on the platform is ordinarily insufficient for learning in a watermaze; however, we have seen that when done in the context of a daily protocol that usually involves swimming to the platform, it seems that the rats do process information about location while on the escape platform even though they have not swum there on that trial (Devan et al. 2002). Such a memory is detectable $30 \mathrm{~min}$ later in a standard swimming probe test, but is weak in the sense that it decays to 
chance within $24 \mathrm{~h}$. The intended modulation event would be three spaced swim trials, in a separate watermaze, the supposition being that as this protocol reliably produces 24 -h memory, such trials must up-regulate PRPs that stabilize synaptic change. A further paper in this series will present these data, together with the impact of our behavioral protocols on the up-regulation of immediate early genes.

\section{Conclusions}

The key results of this series of experiments are that an "everyday" spatial memory paradigm with repeated memory encoding and a search preference measure is possible in the watermaze, and that this produces a quantitatively reliable and monotonic forgetting over a 24 -h period. This protocol shows sensitivity to standard parameters, such as number and spacing of encoding trials, and we have separately shown that it is also more sensitive to hippocampal lesion and pharmacological manipulations than the original paradigm relying exclusively on latency and path-length measures (Bast et al. 2009; Pezze and Bast 2012). Due to this sensitivity, this new DMP paradigm involving a search preference measure could also be of translational value in studies of the impact of neuromodulatory and cognitive enhancing drugs on memory.

\section{Materials and Methods}

\section{Subjects}

A total of 54 adult male Lister Hooded rats (Charles River, Margate, UK) were used as subjects (Experiment $1=20$, Experiment $2=18$, Experiments 3 and $4=16$ ). They weighed $220-250 \mathrm{~g}$ at the start of the experiments, and were housed two per cage in a temperaturecontrolled $\left(20^{\circ} \mathrm{C}-23^{\circ} \mathrm{C}\right)$ and humidity-controlled $(40 \%-55 \%)$ room with an artificial light/dark cycle (lights on 700 a.m. to 7:00 p.m.) and maintained on ad libitum food and water. The rats were transported to, and kept in, the watermaze room in separate cages (two rats at a time). All rats were habituated to handling by the experimenter before the start of the experiments ( 5 d, $\sim 2$ min per rat each day). All experimental procedures were conducted during the light phase of the cycle. The work was conducted under the auspices of a UK Home Office Licence for animal experimentation held by R.G.M.M.

\section{Apparatus}

Training was conducted using a watermaze (circular pool, 2-m diameter, $60-\mathrm{cm}$ height) containing water at $25^{\circ} \mathrm{C} \pm 1^{\circ} \mathrm{C}$ made opaque by the addition of $200 \mathrm{~mL}$ of latex liquid (CementoneBeaver Ltd). The water was changed daily using an automatic filling and draining system. The watermaze was located in a well-lit room containing prominent extra-pool visual cues (e.g., white curtains collected together at one point of the pool [SE], metal racks, and posters on the wall). No cues were located within the pool. To start a trial, rats were released from one of four start positions $(\mathrm{N}, \mathrm{E}, \mathrm{S}, \mathrm{W})$ around the pool. The rats' only escape route from the water was via a single escape platform of 12 -cm diameter. The platform was hidden $1-2 \mathrm{~cm}$ below the water surface. We used a so-called Atlantis platform (Spooner et al. 1994), which can be withheld at $>30 \mathrm{~cm}$ below the water surface by a computercontrolled electromagnet for a predetermined time, making it inaccessible to the rats, before rising to its normal position. This allowed us to run probe trials during which the animals' search preference for the zone containing the platform location was monitored during the first $60 \mathrm{sec}$, before the platform was made available for escape and so reinforce spatially focused searching. The rats' swimming behavior was monitored via a system of video cameras connected to a computer in a control room adjacent to the watermaze room. The computer ran custom-written Watermaze software (Actimetrics and Watermaze Software) that digitizes the path taken by the rats and computes various behavioral measures.

\section{Training}

Trials began at N, W, S, or E in a pseudorandom sequence, with the rats facing the side-walls. Notional inner and outer "rings" were defined for distribution of the escape platforms $(40$ and $70 \mathrm{~cm}$ away from the center of the pool, respectively) with a total of eight locations (compare Fig. 1B). Rats were given different sequences of platform locations (that were counterbalanced across the different conditions), equally distributed across sequences and days, and never repeated within the same batch of rats (see specifications below). Rats were allowed a maximum of 2 min to find the platform and $30 \mathrm{sec}$ on the platform after escape (except for one condition in Experiment 2 where the time on the platform was reduced to 6 sec in order to examine the effect this may have on place learning). If a rat failed to escape within $2 \mathrm{~min}$, it was guided to the platform by the experimenter.

\section{DMP task with search preference measure}

In this new version of the DMP task, the retention trial (probe trial) is altered so that the Atlantis platform is only made available after $60 \mathrm{sec}$ (Fig. 1A). This allows analyzing the proportion of time the rats spend searching the zone where the platform was located on the previous trial(s). The zone analysis compared the time rats spent swimming in the correct platform zone (area defined by a $20-\mathrm{cm}$ radius from the center of the platform) to the time spent in seven other equally sized zones (Fig. 1B). The correct platform zone and the seven additional zones were distributed symmetrically over the pool and were nonoverlapping. The specific set of eight platform positions analyzed on a given probe was defined by the location of the platform at encoding. The zone analysis was calculated as follows: ([time in correct zone/total time in all eight zones] $\times 100$ ). Probe trial latencies corresponded to the time rats took to intersect the area where the platform was located (crossing latencies). Latency savings were calculated as the difference in latencies obtained in the first encoding trial of the day and in the retention trial.

Platform positions were counterbalanced such that they were equally distributed across sequences, days, and retention intervals tested. Two different sequences of platform positions were used in each experiment (including training and probe days). Each sequence comprised inner (I) and outer (O) ring positions. Sequences were designed so that all transitions I-O, O-I, I-I, and $\mathrm{O}-\mathrm{O}$ were equally present and that no learning rule could be established on the basis of the inner or outer location of the platform. Probe trials started with a pseudorandom sequence of start positions. Daily start positions were adjusted between conditions with one, three, or six encoding trials so that (1) positions were always the same for the retention trial and its preceding trial and (2) the starting position for the retention trial was not used during the encoding trials (e.g., one trial conditions [N-SEW], three trial conditions $[\mathrm{EWN}-\mathrm{S}]$, six trial conditions [EWNEWN-S]].

\section{Within-subject experimental designs}

\section{Experiment 1: replication 1 (16 d)}

The rats were allocated to four sequence groups (throughout replications 1-3) and given an initial block of eight training days to eight symmetrically distributed platform positions, counterbalanced across groups and days. In the first four training days, the rats were trained with a 15 -sec interval between trials 1 and 2 . Thereafter, the interval was varied to familiarize rats with the different retention intervals used in replication $1,15 \mathrm{sec}, 1 \mathrm{~h}, 3$ $\mathrm{h}$, and $6 \mathrm{~h}$. Each group of rats was tested at a different retention interval each day. The daily order of testing was counterbalanced with respect to retention intervals between days, with each cohort of rats moved from the rat room to the watermaze room for testing accordingly, one at a time. Rats were kept in the watermaze room during the retention interval, including at intervals of $>15 \mathrm{sec}$ during which they were placed under the watermaze (which was on a raised platform).

After the initial training, the rats were given a sequence of four probe days, each one of them preceded by a training day 
with the same retention interval. Each probe day tested the same group of rats for a different retention interval. The four groups of rats were tested at different retention intervals within a probe day. Eight new symmetrically distributed platform positions were used, which were counterbalanced across groups, days, and ITIs between probe days and between training days. As in training days $5-8$, the rats were moved from day 9 onward into the watermaze room and kept there throughout the duration of daily testing.

\section{Experiment 1: replication 2 (8 d)}

This replication was conducted as on days 9 to 16 of replication 1 , the difference being retention intervals tested, sequence of start positions, and platform positions.

\section{Experiment 1: replication 3 (6 d)}

Testing was at retention intervals of $6 \mathrm{~h}$ and $24 \mathrm{~h}$. The use of a $24-\mathrm{h}$ interval required the use of $2 \mathrm{~d}$ per probe. Over $6 \mathrm{~d}$, there were two probes, each preceded by a training day. On training days, a retention interval of $15 \mathrm{sec}$ was used. On the first day of a probe, half of the rats were tested for a retention interval of $6 \mathrm{~h}$ and the other half was given a single encoding trial. Memory for the location of the platform for that single trial was tested $24 \mathrm{~h}$ later. Four sequences of four different platform positions were used in this series. Platform positions were counterbalanced between training days for sequence groups and days, and between probes for sequence groups, days, and retention intervals. Unlike series 1 and 2 , the rats were not kept in the watermaze room during the retention interval, as it was impossible to retain rats in the watermaze room overnight. Rats were moved back to the vivarium after the encoding trials.

\section{Experiment 2 (28 d)}

As there are six conditions in the study (see Fig. 2A), the rats were allocated to six sequences of platform positions (so that all conditions could be tested on each probe day). During an initial block of four training days the ITI between trials 1 and 2 was $15 \mathrm{sec}$. This ITI was increased to $6 \mathrm{~h}$ on the remaining six training days to familiarize the rats with the retention interval assessed on probe days. In addition, days 7 and 10 included probe trials to establish that performance at $6 \mathrm{~h}$ had reached asymptotic levels before testing (data not shown). This was followed by a block of six probe days with interspersed training days. Each probe day was preceded by two training days with a T1-T2 ITI of $6 \mathrm{~h}$. The main purpose of the training days was to prevent carry over effects between probe days and to assess the stability of performance throughout the experiment. Since memory strength at $6 \mathrm{~h}$ did not vary between replication 1 (in which rats were kept in the watermaze during the retention interval) and 3 (in which rats were moved back to the vivarium; see Results) of Experiment 1, we returned the rats to the vivarium after encoding during Experiments $2-4$.

\section{Experiment 3: series 1 (16 d)}

The animals were given an initial block of four training days with a retention interval of $15 \mathrm{sec}$. This was followed by two replications of two probe days, each probe day preceded by two training days (6-h retention interval). Two sequences of 16 platform positions were used that were counterbalanced for groups, days, and conditions in probe days.

\section{Experiment 3: series 2 (6 d)}

Rats were given a single block of two probe days, each probe day preceded by two training days (15-sec retention interval), with training conditions otherwise identical to series 1 .

\section{Experiment 4 (20 d)}

This experiment used the same rats as tested in Experiment 3. The rats had not been trained for several weeks so they were given four "reminder" training days (four trials/day, 15-sec ITI) to reestablish levels of performance prior to testing (data not shown). After this, performance for the different experimental conditions was assessed in a series of four probe tests (swim vs. placement, $30 \mathrm{~min}$ vs. $24 \mathrm{~h}$ ). Each probe comprised $2 \mathrm{~d}$ (because of the 24 -h retention interval) and was preceded by two training days (four trials/day, 15-sec ITI).

\section{Statistical analysis}

Repeated-measures analysis of variance (ANOVA) was used to examine the impact of within-subjects variables on behavioral measures. Fisher's least significant difference (LSD) test was used to further examine main effects of the ANOVA. Two-tailed onesample $t$ tests were used to compare search preference measures to the value expected by chance (12.5\%). The percent relative variability of the different measures of performance was calculated as the standard error of the mean divided by the absolute value of the mean and multiplied by $100 \%$. Differences in the relative variability of the performance measures were analyzed with a factorial ANOVA. For comparison of the watermaze and event arena data, normalization of the watermaze data to chance was calculated as the percentage of time swimming in the correct zone divided by chance level (12.5\%). Normalization of the event arena data to chance was calculated as the percentage of time digging in the correct sandwell divided by chance level (20\%). The level of significance was set $P<0.05$. Data are presented as mean \pm 1 SEM.

\section{Acknowledgments}

This work was supported by a $\mathrm{PhD}$ scholarship from the Portuguese Fundação para a Ciência e Tecnologia (B.M.d.S.); a Medical Research Council Programme Grant and European Advanced Investigator Grant (R.G.M.M.); and a Royal Society Research Grant and Caledonian Research Foundation Personal Fellowship (T.B.). We are grateful to Patrick Spooner for computer assistance and Richard Watson for care of the animals.

\section{References}

Aggleton JP, Pearce JM. 2001. Neural systems underlying episodic memory: Insights from animal research. Philos Trans R Soc Lond B Biol Sci 356: $1467-1482$.

Aggleton JP, O'Mara SM, Vann SD, Wright NF, Tsanov M, Erichsen JT. 2010. Hippocampal-anterior thalamic pathways for memory: Uncovering a network of direct and indirect actions. Eur I Neurosci 31: 2292-2307.

Aguilar-Valles A, Sanchez E, de Gortari P, Balderas I, Ramirez-Amaya V, Bermudez-Rattoni F, Joseph-Bravo P. 2005. Analysis of the stress response in rats trained in the water-maze: Differential expression of corticotropin-releasing hormone, CRH-R1, glucocorticoid receptors and brain-derived neurotrophic factor in limbic regions. Neuroendocrinology 82: 306-319.

Ballarini F, Moncada D, Martinez MC, Alen N, Viola H. 2009. Behavioral tagging is a general mechanism of long-term memory formation. Proc Natl Acad Sci 106: 14599-14604.

Bast T, da Silva BM, Morris RG. 2005. Distinct contributions of hippocampal NMDA and AMPA receptors to encoding and retrieval of one-trial place memory. J Neurosci 25: 5845-5856.

Bast T, Wilson IA, Witter MP, Morris RG. 2009. From rapid place learning to behavioral performance: A key role for the intermediate hippocampus. PLoS Biol 7: e1000089.

Bolding K, Rudy JW. 2006. Place learning in the Morris water task: Making the memory stick. Learn Mem 13: 278-286.

Bolhuis JJ, Buresova O, Bures J. 1985. Persistence of working memory of rats in an aversively motivated radial maze task. Behav Brain Res 15: 43-49.

Buresova O, Krekule I, Zahalka A, Bures J. 1985. On-demand platform improves accuracy of the Morris water maze procedure. J Neurosci Methods 15: 63-72.

Chen G, Chen KS, Knox J, Inglis J, Bernard A, Martin SJ, Justice A, McConlogue L, Games D, Freedman SB, et al. 2000. A learning deficit related to age and $\beta$-amyloid plaques in a mouse model of Alzheimer's disease. Nature 408: 975-979. 
de Hoz L, Moser EI, Morris RG. 2005. Spatial learning with unilateral and bilateral hippocampal networks. Eur J Neurosci 22: 745-754.

de Quervain DJ, Roozendaal B, McGaugh JL. 1998. Stress and glucocorticoids impair retrieval of long-term spatial memory. Nature 394: $787-790$.

Devan BD, Petri HL, Mishkin M, Stouffer EM, Bowker JL, Yin PB, Buffalari DM, Olds JL. 2002. A room with a view and a polarizing cue: Individual differences in the stimulus control of place navigation and passive latent learning in the water maze. Neurobiol Learn Mem 78: 79-99.

Domjan M. 1980. Effects of the intertrial interval on taste-aversion learning in rats. Physiol Behav 25: 117-125.

Ebbinghaus H. 1885. Über das gedächtnis: Untersuchungen zur experimentellen psychologie. Duncker \& Humblot, Leipzig, Germany.

Eichenbaum H. 2000. A cortical-hippocampal system for declarative memory. Nat Rev Neurosci 1: 41-50.

Euston DR, Gruber AJ, McNaughton BL. 2012. The role of medial prefrontal cortex in memory and decision making. Neuron 76: 1057-1070.

Fanselow MS, Tighe TJ. 1988. Contextual conditioning with massed versus distributed unconditional stimuli in the absence of explicit conditional stimuli. J Exp Psychol Anim Behav Process 14: 187-199.

Ferbinteanu J, Ray C, McDonald RJ. 2003. Both dorsal and ventral hippocampus contribute to spatial learning in Long-Evans rats. Neurosci Lett 345: 131-135.

Frey U, Morris RG. 1998. Synaptic tagging: Implications for late maintenance of hippocampal long-term potentiation. Trends Neurosci 21: $181-188$.

Frey U, Huang YY, Kandel ER. 1993. Effects of cAMP simulate a late stage of LTP in hippocampal CA1 neurons. Science 260: 1661-1664.

Genoux D, Haditsch U, Knobloch M, Michalon A, Storm D, Mansuy IM. 2002. Protein phosphatase 1 is a molecular constraint on learning and memory. Nature 418: 970-975.

Jackson SJ, Hussey R, Jansen MA, Merrifield GD, Marshall I, MacLullich A, Yau JL, Bast T. 2011. Manganese-enhanced magnetic resonance imaging (MEMRI) of rat brain after systemic administration of $\mathrm{MnCl}_{2}$ : Hippocampal signal enhancement without disruption of hippocampus-dependent behavior. Behav Brain Res 216: 293-300.

Jacobs LF, Schenk F. 2003. Unpacking the cognitive map: The parallel map theory of hippocampal function. Psychol Rev 110: 285-315.

Josselyn SA, Shi C, Carlezon WA Jr., Neve RL, Nestler EJ, Davis M. 2001. Long-term memory is facilitated by cAMP response element-binding protein overexpression in the amygdala. J Neurosci 21: 2404-2412.

Keith JR, McVety KM. 1988. Latent place learning in a novel environment and the influences of prior training in rats. Psychobiology 16: 146-151.

Kogan JH, Frankland PW, Blendy JA, Coblentz J, Marowitz Z, Schutz G, Silva AJ. 1997. Spaced training induces normal long-term memory in CREB mutant mice. Curr Biol 7: 1-11.

Leutgeb S, Leutgeb JK, Moser MB, Moser EI. 2005. Place cells, spatial maps and the population code for memory. Curr Opin Neurobiol 15: 738-746.

Lisman JE, Grace AA. 2005. The hippocampal-VTA loop: controlling the entry of information into long-term memory. Neuron 46: 703-713.

Lisman J, Grace AA, Duzel E. 2011. A neoHebbian framework for episodic memory; role of dopamine-dependent late LTP. Trends Neurosci 34: $536-547$.

Luksys G, Gerstner W, Sandi C. 2009. Stress, genotype and norepinephrine in the prediction of mouse behavior using reinforcement learning. Nat Neurosci 12: 1180-1186.

McGaugh JL. 1966. Time-dependent processes in memory storage. Science 153: $1351-1358$.

Moncada D, Viola H. 2007. Induction of long-term memory by exposure to novelty requires protein synthesis: Evidence for a behavioral tagging. $J$ Neurosci 27: 7476-7481.

Moncada D, Ballarini F, Martinex MC, Frey JUL, Viola H. 2011 Identification of transmitter systems and learning tag molecules involved in behavioral tagging during memory formation. Proc Natl Acad Sci 108: 12931-12936.

Morris RG. 1981. Spatial localization does not require the presence of local cues. Learn Motiv 12: 239-260.

Morris RG. 1983. An attempt to dissociate spatial-mapping and working-memory theories of hippocampal function. In The neurobiology of the hippocampus (ed. Siefert W), pp. 405-432. Academic Press, London.

Morris RG. 1984. Developments of a water-maze procedure for studying spatial learning in the rat. J Neurosci Methods 11: 47-60.

Morris RG, Doyle J. 1985. Successive incompatible tasks: Evidence for separate subsystems for storage of spatial knowledge. In Electrical activity of the archicortex (ed. Buzsaki G, Vanderwolf CH), pp. 281-293. Akademiai Kiado, Budapest, Hungary.

Morris RG, Garrud P, Rawlins JN, O'Keefe J. 1982. Place navigation impaired in rats with hippocampal lesions. Nature 297: 681-683.

Morris RG, Schenk F, Tweedie F, Jarrard LE. 1990. Ibotenate lesions of hippocampus and/or subiculum: Dissociating components of allocentric spatial learning. Eur J Neurosci 2: 1016-1028.
Moser EI, Krobert KA, Moser MB, Morris RG. 1998. Impaired spatial learning after saturation of long-term potentiation. Science 281: 2038-2042.

Nakazawa K, Sun LD, Quirk MC, Rondi-Reig L, Wilson MA, Tonegawa S. 2003. Hippocampal CA3 NMDA receptors are crucial for memory acquisition of one-time experience. Neuron 38: 305-315.

O'Carroll CM, Martin SJ, Sandin J, Frenguelli B, Morris RG. 2006. Dopaminergic modulation of the persistence of one-trial hippocampus-dependent memory. Learn Mem 13: 760-769.

O'Keefe J. 1976. Place units in the hippocampus of the freely moving rat. Exp Neurol 51: 78-109.

O'Keefe J, Nadel L. 1978. The hippocampus as a cognitive map. Clarendon Press, Oxford.

Panakhova E, Buresova O, Bures J. 1984. Persistence of spatial memory in the Morris water tank task. Int J Psychophysiol 2: 5-10.

Pezze M, Bast T. 2012. Dopaminergic modulation of hippocampus-dependent learning: Blockade of hippocampal D1-class receptors during learning impairs 1 -trial place memory at a 30-min retention delay. Neuropharmacology 63: 710-718.

Redondo RL, Morris RG. 2011. Making memories last: The synaptic tagging and capture hypothesis. Nat Rev Neurosci 12: 17-30.

Rescorla RA. 1989. Behavioral studies of Pavlovian conditioning. Ann Rev Neurosci 11: $329-352$.

Reymann KG, Malisch R, Schulzeck K, Brodemann R, Ott T, Matthies H. 1985. The duration of long-term potentiation in the CA1 region of the hippocampal slice preparation. Brain Res Bull 15: 249-255.

Roberts WA, Dale RHI. 1981. Remembrance of places lasts: Proactive inhibition and patterns of choice in rat spatial memory. Learn Motiv 12: 261-281.

Scharf MT, Woo NH, Lattal KM, Young JZ, Nguyen PV, Abel T. 2002. Protein synthesis is required for the enhancement of long-term potentiation and long-term memory by spaced training. I Neurophysiol 87: 2770-2777.

Schenk F, Morris RG. 1985. Dissociation between components of spatial memory in rats after recovery from the effects of retrohippocampal lesions. Exp Brain Res 58: 11-28.

Sisti HM, Glass AL, Shors TJ. 2007. Neurogenesis and the spacing effect: Learning over time enhances memory and the survival of new neurons. Learn Mem 14: 368-375.

Spooner RI, Thomson A, Hall J, Morris RG, Salter SH. 1994. The Atlantis platform: A new design and further developments of Buresova's on-demand platform for the water maze. Learn Mem 1: 203-211.

Spreng M, Rossier J, Schenk F. 2002. Spaced training facilitates long-term retention of place navigation in adult but not in adolescent rats. Behav Brain Res 128: 103-108.

Squire LR. 1992. Memory and the hippocampus: A synthesis from findings with rats, monkeys, and humans. Psychol Rev 99: 195-231.

Steele RJ, Morris RG. 1999. Delay-dependent impairment of a matching-to-place task with chronic and intrahippocampal infusion of the NMDA-antagonist D-AP5. Hippocampus 9: 118-136.

Strijkstra AM, Bolhuis JJ. 1987. Memory persistence of rats in a radial maze varies with training procedure. Behav Neural Biol 47: 158-166.

Sutherland RJ, Linggard R. 1982. Being there: A novel demonstration of latent spatial learning in the rat. Behav Neural Biol 36: 103-107.

Sutherland RJ, Rodriguez AJ. 1989. The role of the fornix/fimbria and some related subcortical structures in place learning and memory. Behav Brain Res 32: 265-277.

Sutherland RJ, Whishaw IQ, Kolb B. 1983. A behavioural analysis of spatial localization following electrolytic, kainate- or colchicine-induced damage to the hippocampal formation in the rat. Behav Brain Res 7: 133-153.

Sutherland RJ, Whishaw IQ, Kolb B. 1988. Contributions of cingulate cortex to two forms of spatial learning and memory. J Neurosci 8: 1863-1872.

von Linstow Roloff E, Platt B, Riedel G. 2002. Long-term study of chronic oral aluminum exposure and spatial working memory in rats. Behav Neurosci 116: 351-356.

Wang SH, Morris RG. 2010. Hippocampal-neocortical interactions in memory formation, consolidation, and reconsolidation. Annu Rev Psychol 61: 49-79, C41-C44.

Wang SH, Redondo RL, Morris RG. 2010. Relevance of synaptic tagging and capture to the persistence of long-term potentiation and everyday spatial memory. Proc Natl Acad Sci 107: 19537-19542.

Whishaw IQ. 1985. Formation of a place learning-set by the rat: A new paradigm for neurobehavioral studies. Physiol Behav 35: 139-143.

Whishaw IQ. 1991. Latent learning in a swimming pool place task by rats: Evidence for the use of associative and not cognitive mapping processes. Q J Exp Psychol 43B: 83-103.

Wise RA. 2004. Dopamine, learning and motivation. Nat Rev Neurosci 5: $483-494$.

Received June 17, 2013; accepted in revised form October 6, 2013. 


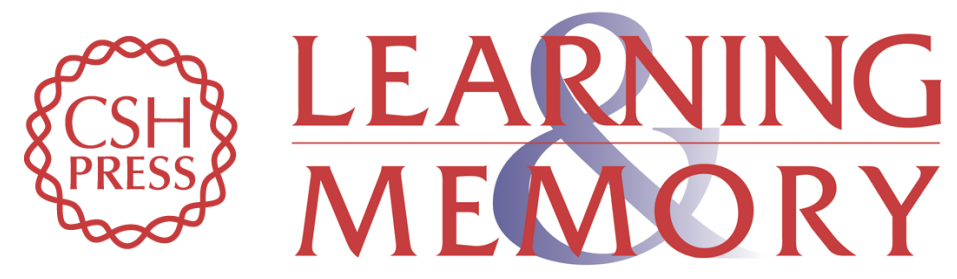

\section{Spatial memory: behavioral determinants of persistence in the watermaze delayed matching-to-place task}

Bruno M. da Silva, Tobias Bast and Richard G.M. Morris

Learn. Mem. 2014, 21:

Access the most recent version at doi:10.1101//m.032169.113

\begin{aligned} & \hline References $\begin{array}{l}\text { This article cites } 65 \text { articles, } 14 \text { of which can be accessed free at: } \\ \text { http://learnmem.cshlp.org/content/21/1/28.full.html\#ref-list-1 }\end{array} \\ & \begin{array}{r}\text { Creative } \\ \text { Commons } \\ \text { License }\end{array} \begin{array}{l}\text { This article is distributed exclusively by Cold Spring Harbor Laboratory Press for the } \\ \text { first } 12 \text { months after the full-issue publication date (see } \\ \text { http://learnmem.cshlp.org/site/misc/terms.xhtml). After } 12 \text { months, it is available under } \\ \text { a Creative Commons License (Attribution-NonCommercial 3.0 Unported), as } \\ \text { described at http://creativecommons.org/licenses/by-nc/3.0/. }\end{array} \\ & \begin{array}{c}\text { Receive free email alerts when new articles cite this article - sign up in the box at the } \\ \text { top right corner of the article or click here. }\end{array} \\ &$ Service \end{aligned} 